\title{
Corrigendum
}

\section{Whole-grain consumption and the risk of all-cause, CVD and cancer mortality: a meta-analysis of prospective cohort studies - CORRIGENDUM}

Honglei Wei, Zong Gao, Rui Liang, Zengqiang Li, Hong Hao and Xu Liu

(First published online 20 June 2016)

DOI: http://dx.doi.org/10.1017/S0007114516001975

The address for W.H.L., L.R., L.Z.Q., and L.X. should be "a1 Department of Sports, Southwest Jiaotong University, Chengdu, Sichuan Province, 611756, China" instead of "al Department of Sports, Jiaotong University, Chengdu, Sichuan Province, 611756, China".

\section{Reference}

1. Wei H, Gao Z, Liang R, et al. (2016) Whole-grain consumption and the risk of all-cause, CVD and cancer mortality: a meta-analysis of prospective cohort studies. Br J Nutr 116, 514-525. doi:10.1017/S0007114516001975. 